\section{Principles and Practice of Clinical Research}

A Global Journal in Clinical Research

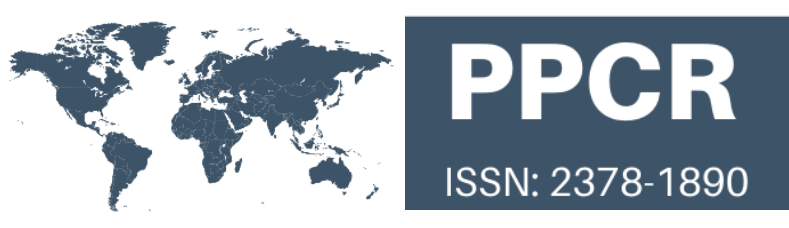

Supplementation of curcumin and piperine plus standard therapy to prevent exacerbations in patients with Chronic Obstructive Pulmonary Disease (CUPISCO TRIAL: CUrcumin and Plperine Supplementation for COpd). A study protocol.

D. Lukac ${ }^{1 \star \#}$, E. Martínez-Lozano2\#, K. Vásquez-Ávila ${ }^{3 \#, ~ M . ~ A l ~ D o s o u k y ~}{ }^{4}$ A. Francone ${ }^{5}$, J. GómezFarías $^{6}$, K. Lee ${ }^{7}$, R. Mendes ${ }^{8}$, D. Mahmoud ${ }^{9}$, I. Montes-Posada ${ }^{10}$, L. Okada ${ }^{11}$, B. Schembida de Oliveira $^{12}$, D. Raglione ${ }^{13}$, R. Visco-Costa ${ }^{14}$.

\#These authors have contributed equally to this work.

*Corresponding author: Danitza Lukac, Facultad de Medicina Humana, Universidad San Martín de Porres, Lima, Perú. Email: danitza.lukac@gmail.com

Rest of author's affiliation at the end of the manuscript.

Received December 23, 2020; accepted February 15, 2021; published February 25, 2021.

\begin{abstract}
:
Background: Chronic Obstructive Pulmonary Disease (COPD) is a highly prevalent condition that leads to a variety of complications that increase social and economic burdens. Although current treatment guidelines target symptomatic relief, including strategies to reduce future exacerbations and improve patients' quality of life, there is still a need for an effective intervention that also reduces the healthcare cost associated with this disease through reducing exacerbationrelated hospitalization. Curcumin and piperine are natural and inexpensive components proven to inhibit the main inflammatory cascade responsible for COPD exacerbations.

Objective: This trial aims to evaluate the supplementation of curcumin and piperine with COPD standard therapy to reduce the exacerbation rate in COPD patients compared to standard therapy alone.

Methods: This will be a phase II multi-center, randomized, placebo-controlled, triple-blinded, parallel-group trial with COPD patients, classified in GOLD C and D groups, who are currently under standard treatment. Supplementation with $4 \mathrm{~g}$ of curcumin combined with $20 \mathrm{mg}$ of piperine versus placebo will be evaluated during a period of 12 months. One thousand seven hundred and sixteen participants will be enrolled, with 858 participants for the control and intervention groups. The primary outcome will be the mean rate of moderate and severe exacerbations. Secondary outcomes will include time until first moderate or severe COPD exacerbation, St. George's Respiratory Questionnaire response, clinically significant change in post-bronchodilator forced expiratory volume, modification in the GOLD groups, and the presence of adverse events.

Discussion: This will be the first randomized clinical trial to test curcumin plus piperine in COPD patients for twelve months. This intervention may improve patients' quality of life and reduce the economic and social burden of this disease.
\end{abstract}

Keywords: COPD, exacerbation, curcumin, piperine

DOI: http://dx.doi.org/10.21801/ppcrj.2020.64.4 


Abbreviations:
CTCAE: Common Terminology Criteria for Adverse
Events
COPD: chronic obstructive pulmonary disease
FEV1: forced expiratory volume in 1 second
FVC: forced vital capacity
GOLD: Global initiative for chronic Obstructive Lung
Disease
SGRQ: St. George's Respiratory Questionnaire

\section{INTRODUCTION}

Chronic Obstructive Pulmonary Disease (COPD) is a group of life-threatening chronic conditions characterized by progressive airflow limitation. The Global Initiative for Chronic Obstructive Lung Disease Pocket Guide (GOLD) states that COPD is the fourth leading cause of death worldwide, affecting $13,1 \%$ of the world population. In the United States, COPD affects more than 16 million people (Blanco et al., 2019) and accounts for costs of approximately 52.4 billion dollars annually, especially due to exacerbations, which represent the main financial burden (Guarascio et al., 2013). Currently, management includes symptomatic relief and strategies for risk reduction of future exacerbations. The interest lies in the prevention of exacerbations by using long-acting beta-agonists, longacting muscarinic antagonists, inhaled corticosteroids, mucolytics, vaccinations, and long-term macrolides (Ko et al., 2016), which so far have not shown a significant decrease in mortality rates (Agusti et al., 2014).

COPD pathophysiology involves an excessive inflammatory response, resulting from the inhalation of harmful environmental particles and gases. Subsequent accumulation of multiple inflammatory cells including macrophages, neutrophils, and $\mathrm{T}$ lymphocytes (especially CD8) leads to the release of toxic mediators, capable of progressively damage the lung parenchyma (GOLD, 2020). Therefore, new experimental drugs are being investigated to disrupt this inflammatory response. One of these new components is curcumin, a phytochemical compound present in turmeric, a plant from the ginger family. In COPD preclinical models, curcumin has been associated with the reduction of inflammatory molecules including nuclear factor kappa B, tumor necrosis factor, interleukin 6, among others (Prasad, Tyagi \& Aggarwal, 2014; Biswas et al., 2005; Panahi et al., 2016). Moreover, it has been hypothesized that COPD patients with glucocorticoid resistance could benefit from curcumin supplementation since curcumin restores histone deacetylase activity, a nuclear enzyme found to be diminished in these patients (Meja et al., 2008)

Curcumins' previous studies have shown few side effects and demonstrated a high safety margin. However, curcumin undergoes rapid metabolic biotransformation, reducing its serum concentration, and increasing renal elimination (Lao et al., 2006). Therefore, new formulations have been manufactured to improve curcumin's pharmacokinetics (Sasaki et al., 2011; Prasad et al., 2014). Shoba et al. (1998) demonstrated the use of piperine to enhance the serum concentration, absorption, and overall bioavailability of curcumin in both rats and healthy human volunteers with no adverse effects.

There is still a need to improve COPD treatment, to find a novel and effective intervention that also reduces the healthcare costs associated with this disease by reducing exacerbation-related hospitalization. Despite the proven antioxidant and anti-inflammatory properties of curcumin, to our knowledge, there are no studies of its combination with piperine to treat COPD. This randomized trial protocol aims to evaluate these natural, non-toxic components in an attempt to reduce future exacerbations in patients classified as GOLD C \& D.

\section{MATERIAL AND METHODS}

\section{Trial design}

This is a phase II randomized, placebo-controlled, tripleblinded, parallel-group, and multi-center trial of COPD patients in GOLD groups C and D with a primary endpoint of the mean rate of moderate to severe COPD exacerbations after 12 months follow-up.

\section{Study setting}

The study will be conducted in three COPD referral centers located in different regions of the United States of America.

\section{Randomization}

Participants will be recruited by each center. Blocked randomization, in a 1:1 ratio, will be performed using an online randomization service (TENALEA) with permuted blocks of four, six, and eight subjects, releasing the randomization code after the recruitment of the patient and all baseline measurements are finalized. Data from all eligible participants who consent will be entered into the web database by the staff 
member assigned for recruitment and clinical interviews.

\section{Blinding}

This will be a triple-blinded study in which subjects, attending physicians, and outcome assessors/statisticians will be unaware of allocation. Assigned pharmacists will have unblinded access to the randomization code.

Because curcumin and piperine have noticeable tastes and colors, both intervention and placebo capsules will be similar in size, color, consistency, and packaging. Placebo will have an orange-colored starch powder that will have intestinal and urinary elimination to mimic the intervention.

Blinding's efficacy will be measured using Bang's Blinding Index after 50\% enrollment and at trial termination. Unblinding of participants will proceed after study publication.

\section{Emergency unblinding}

In case of severe adverse events, emergency unblinding can take place. The researcher will access the database and fill a form stating why the blinding was broken. Other members of the research team will not be informed. Participants will only be informed of their allocation if needed.

There will be a 24-hour hotline for subjects to report adverse events and participants will be encouraged to call in case they need immediate medical attention.

\section{Eligibility}

Inclusion Criteria:

- $\quad$ Age $\geq 40$;

- Previous COPD diagnosis confirmed by spirometry (FEV1/FVC ratio < 0.7 after bronchodilator);

- Symptoms severity classified as GOLD C or D;

- Exacerbation free period of at least 4 weeks before baseline measurements;

- Able to perform spirometry (American Thoracic Society/European Respiratory Society acceptability criteria);

- Autonomy to consent;

- Available to participate in a 12-month clinical trial.

\section{Exclusion Criteria:}

- Respiratory diseases: asthma, interstitial fibrosis, sarcoidosis, and other occupational lung diseases;

- History of lung resection;
- New York Heart Association functional heart failure classes III \& IV;

- Liver or renal failure;

- Insulin-dependent diabetes;

- Neuropsychiatry conditions: dementia, other neurocognitive deficits, schizophrenia, and bipolar disorders;

- Previous use of systemic corticosteroids within 4 weeks before baseline measurements;

- Use of commercial curcumin supplements within 14 days before baseline measurements;

- Immunosuppressive therapies;

- Malabsorption syndromes;

- Abnormal levels of lactate dehydrogenase/serum alkaline phosphatase;

- Anemia;

- Coagulopathies;

- Use of anticoagulants;

- Pregnancy and breastfeeding;

- Use of drugs metabolized by cytochrome p450;

- Current alcohol dependence or drug abuse;

- Confirmed allergy to any of the components found on curcumin and/or piperine.

\section{Recruitment strategy}

COPD attending clinicians of each site will be contacted in person and by email. Information about the clinical trial will be given, emphasizing the benefits for patients and its clinical relevance. The research teams will share the clinical trial proposal among doctors from other respiratory facilities to enhance trial awareness and improve recruitment. Online advertising will be introduced through each center reference site and google for three months before the trial begins and later on during the recruitment period, if necessary. Online advertisements will actively appear within the Google search window of individuals within a 250 miles radius from each facility who look for the words: "COPD", "Chronic obstructive pulmonary disease", "curcumin", "piperine" or "spirometry".

We will have an ongoing recruitment strategy, in which two investigators will screen medical records for eligibility of COPD patients admitted in the referral centers, 4 hours a day at least 3 times per week. Potential candidates will be presented to the principal investigator of each site.

The informed consent, in English and Spanish, will be approved by the institutional review board of each center. This document will be signed by the subject during the baseline visit. 


\section{Timeline}

\section{CUPISCO Trial Timeline}

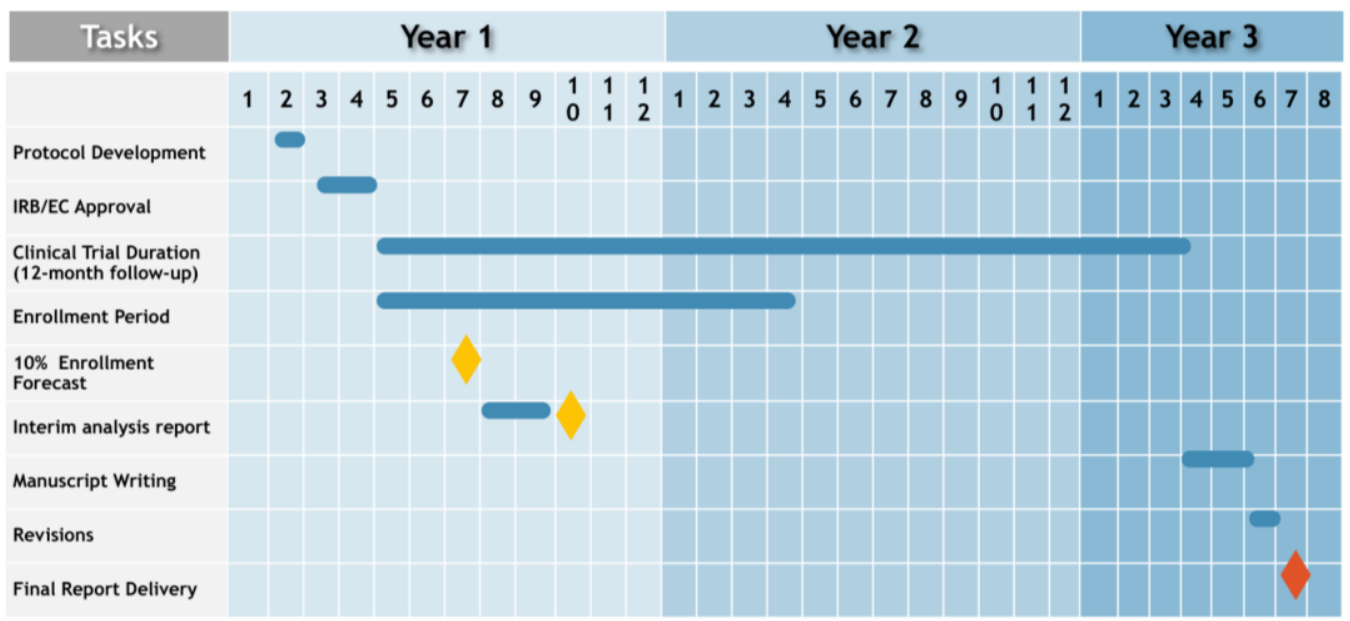

Figure 1. Study timeline of CUPISCO trial

\section{Adherence}

Adherence will be reinforced in every office visit. Before the mandatory appointment, a reminder will be sent via email and/or SMS. In case of missed appointments, reasons will be assessed, and adherence reinforced. After three failed phone calls and email attempts, participants will be excluded from the trial.

At each mandatory visit, participants will be given a flask filled with pills for 3 months and asked to return unused pills, which will be counted to assess adherence. Transportation and food vouchers will be offered on visit days for the subject and a partner.

\section{Intervention}

Different doses of curcumin have been investigated for different diseases, however, a dose of less than $2 \mathrm{~g}$ does not reach the appropriate bioavailability needed for a significant clinical effect (Shoba et al. 1998). The combination of curcumin and $20 \mathrm{mg}$ of piperine has been shown to increase the bioavailability of curcumin by $2000 \%$ (Shoba et al. 1998), without side effects (Panahi et. al 2016). For this reason, a capsule containing $4 \mathrm{~g}$ of curcumin and $20 \mathrm{mg}$ of piperine will be manufactured for this trial, along with a placebo pill containing an artificial colored orange starch. Both pills will share the same characteristics. The intervention will consist of a daily oral administration of the curcumin/piperine pill, or placebo, combined with the standard treatment that each patient was receiving before entering the trial, according to the GOLD Guideline. All treating physicians will receive appropriate training on possible standard treatment combinations accordingly. The first meeting between the physicians and the clinical trial team will take place prior to patient enrollment.

\section{Modification/discontinuation}

No dose modifications will be made during the trial. Curcumin supplementation rarely presents significant adverse events (Cheng et al., 2001). If mild gastrointestinal side effects occur, treatment will be continued, as those often improve spontaneously.

Treatment will be interrupted in cases of adverse events grade 3 or higher according to the Common Terminology Criteria for Adverse Events (CTCAE) version 5.0 (U.S. Department of Health and Human Services (2017)). Luber et al. (2019) reported 2 study cases with induced liver injury related to curcumin (dose of $1125 \mathrm{mg}$ of curcumin). This adverse event is rare, however, a significant increase in liver enzymes without a clear cause would be an indication to stop supplementation. In the first visit, a complete blood count and liver function test will be taken.

\section{Outcomes}

To evaluate the difference in effectiveness between curcumin plus piperine and standard treatment alone, the mean rate of moderate and severe exacerbations 
after a 12-month follow-up will be the primary outcome. Moderate exacerbations are defined as "those leading to treatment with systemic glucocorticosteroids, antibiotics, or both for at least 3 days", and severe, as "resulting in hospitalization or death" (Rabe et al., 2020).

Each patient will be given a diary to record exacerbation events, which will be self-reported at each mandatory visit, every 3 months, and will be corroborated through medical records reviewed by the attending physicians.

If the event occurred in a different health care facility, the patient will be asked to provide a discharge medical record. In the absence of records, the event will not be considered as an exacerbation. An exacerbation will only be counted after being reviewed by the Clinical Event Committee, which will be composed of five blinded pulmonologists not involved in the study.

\section{Secondary outcomes:}

- Time until first moderate or severe COPD exacerbation (since day one of intervention)

- A clinically significant change in postbronchodilator forced expiratory volume (FEV1) $\geq$ 15\% (Cazzola et al., 2008)

- St. George's Respiratory Questionnaire (SGRQ) response with a mean change in score of $\geq 4$ units will be considered slightly efficacious, $\geq 8$ units moderately efficacious and $\geq 12$ very efficacious treatment (Jones, 1994).

The previously listed outcomes will be measured at baseline and every 3 months over a twelve-month period by the attending physician.

Other secondary outcomes are:

- Modification in the GOLD groups (GOLD, 2020), which will be assessed by physicians at baseline and the end of the trial.

- Adverse events will be reported by the patient to the research team and will be monitored throughout the entire trial period. The adverse events assessment will be based on the CTCAE version 5.0 grades (mild, moderate, severe, lifethreatening consequences, death).

\section{Data Management}

Each patient will have a Case Report Form to collect the data filled by the healthcare practitioners during the follow-up appointments. Data will be entered electronically, to the Oracle Clinical database management system, by the Data Coordinating Center, an independent team.

\section{Interim Analysis}

An interim analysis to assess for safety will be performed by the Data and Safety Monitoring Committee when $10 \%$ of the enrollment is complete and those subjects have completed 3 months of followup. The trial will be terminated if $>10 \%$ of participants suffer adverse events grade 3 or higher according to CTCAE version 5.0

\section{Sample size}

Based on previous literature showing a mean exacerbation rate of 1.7 for the control group (Rabe et al, 2020), we designed our study to have $80 \%$ power to detect a reduction of at least $30 \%$ in the mean exacerbation rate (Criner, 2019). The sample size was calculated using the ratio of two negative binomial rates, an effect size of 0.30 , a negative binomial dispersion $(\mathrm{k})$ 1 , alpha 0.05 , and a $20 \%$ dropout rate. We will need to enroll 1716 participants, with 858 participants for the control and intervention groups, respectively.

\section{Statistical Analysis}

Negative binomial regression will be used to assess the mean exacerbation rate at 12 months. Adjustment for potential differences at baseline will be performed for the following covariates: gender, smoking, comorbidities, and age.

For the secondary endpoints, time to first exacerbation will be analyzed using survival analysis, by generating Kaplan-Meier estimates to graphically show exacerbation free time for both groups. Cox proportional-hazard model regression analysis will be performed to assess between-group differences and to adjust for covariates (smoking, age, gender). The change in post-bronchodilator FEV1 will be compared to baseline using a linear mixed model.

St. George's Respiratory Questionnaire (SGRQ), change in the GOLD group, and adverse events will be analyzed using chi-square or Fisher's exact test as appropriate.

All tests will be calculated using 2-sided p-values with an alpha $\leq 0.05$ level of significance. The statistical analyses will be performed using STATA/IC software version 16.1 .

We will use intention to treat (ITT) analysis anticipating that missing data could be fully explained by the association with the observable independent variables. To handle missing data, Stochastic Regression Imputation will be performed. 


\section{DISCUSSION}

A multi-center, triple blinded, randomized, phase II trial will be conducted in COPD patients, classified as GOLD C and D, who are currently under standard treatment. Supplementation with $4 \mathrm{~g}$ of curcumin combined with $20 \mathrm{mg}$ of piperine versus placebo will be used to assess changes in the mean exacerbation rate, a clinically relevant outcome that impacts not only the patients' quality of life but also the entire healthcare system.

The main strength of this study lies in the primary outcome, a hard type endpoint not susceptible to subjectivity. Bias will be limited by large sample size, along with blinding and randomization strategies. Due to the long-term follow-up, the risk of potential placebo effect will be reduced. Strict inclusion criteria will reduce variance in this study population, increasing internal validity. We addressed possible limitations with the study design. First, a reduced effect size extrapolated from past studies led us to increase the sample size. In addition, we decided to do a multi-center trial to increase the feasibility of recruitment, although this could imply less compliance to follow the protocol by center. Second, in a 12-month follow-up, adherence - not only for the intervention but for the follow-up visits - can be challenging. Third, the potential side effects of curcumin as well as the chemical changes it produces in urine may lead to unintentional unblinding of the participants or attending physicians. Finally, dropouts resulting from severe side effects could lead to missing data not at random, threatening the study validity.

This will be the first randomized clinical trial to test curcumin plus piperine in COPD patients for twelve months. Our dosage is based on previous studies, where up to $8 \mathrm{~g}$ of daily curcumin was used for 3 months without adverse events (Cheng et al., 2001). Similarly, the combination of $1.5 \mathrm{~g}$ of curcumin and $20 \mathrm{mg}$ of piperine was safely used for 4 weeks (Panahi et al., 2016). Regardless, we will still assess for safety through an interim analysis.

Previous in vitro studies demonstrating curcumin's anti-inflammatory and antioxidant properties have raised our expectations for this study. However, some factors may negatively affect the outcomes of our trial including curcumin's reduced effect size, insufficient dosage, the sample not being representative of the population, or the personalized treatment combinations received by each patient. Nonetheless, we believe that all of these possibilities, if present, will serve as a guide for future researchers. Negative results could help answer questions and highlight new knowledge gaps that incite researchers to keep pursuing clinical trials in this field.

\section{CONCLUSION}

Curcumin has been part of the human diet and alternative medicine for centuries. Interest in natural remedies is growing, leading to more research about curcumin's properties and its incorporation as a treatment in different inflammatory diseases. We will combine curcumin with piperine to enhance its bioavailability and ensure a dosage capable of showing a clinical benefit. The proposed supplementation presents itself as a safe and inexpensive treatment strategy to improve patients' quality of life and reduce the economical and social burden of this disease.

\section{Author affiliations} 1. Facultad de Medicina Humana, Universidad San Martín de
Porres, Lima, Perú
2. Musculoskeletal Translational Innovation Initiative, Carl J.
Shapiro Department of Orthopaedic Surgery, Beth Israel
Deaconess Medical Center and Harvard Medical School, Boston,
MA, USA
3. Facultad de Medicina, Pontificia Universidad Javeriana,
Bogotá, Colombia
4. Acute Care Surgery, Hamad Medical Corporation, Doha,
Qatar
5. Centro Oftalmológico Dr Charles, Buenos Aires, Argentina
6. Radioneurosurgery Unit, The National Institute of Neurology
and Neurosurgery, Mexico City, Mexico
7. Clinical Affairs Department, Establishment Labs, Alajuela,
Costa Rica
8. Centro de Oncologia, Hospital Sírio-Libanês, Brasília, Brazil
9. Laboratory Department, PHCC Qatar, Doha, Qatar
10. Head of Physical Medicine and Rehabilitation Department,
Doctor Negrin University Hospital, Las Palmas de Gran Canaria,
Spain
11. Cirurgia de cabeça e pescoço, Hospital das Clínicas da
Faculdade de Medicina da Universidade de São Paulo, São
Paulo, Brazil
12. Departamento de Neurocirurgia, Hospital de Clínicas
Municipal José de Alencar, São Paulo, Brazil
13. Instituto do Câncer do Estado de São Paulo, Hospital das
Clínicas da Faculdade de Medicina da Universidade de São
Paulo, São Paulo, Brazil
14. Faculdade de medicina, Universidade Salvador (UNIFACS),
Salvador, Bahia, Brazil

\section{Conflict of interest}

None.

\section{REFERENCES}

Agustí A, Calverley P, Decramer M, Stockley R, Wedzicha J. Prevention of Exacerbations in Chronic Obstructive Pulmonary Disease: Knowns and Unknowns. Chronic Obstr Pulm Dis J COPD Found. Published online 2014. doi:10.15326/jcopdf.1.2.2014.0134 
Biswas, S. K., McClure, D., Jimenez, L. A., Megson, I. L., \& Rahman, I. (2005). Curcumin induces glutathione biosynthesis and inhibits NF-kappaB activation and interleukin-8 release in alveolar epithelial cells: mechanism of free radical scavenging activity. Antioxidants \& redox signaling, 7(1-2), 32-41. https://doi.org/10.1089/ars.2005.7.32

Blanco, I., Diego, I., Bueno, P., Casas-Maldonado, F., \& Miravitlles, M. (2019). Geographic distribution of COPD prevalence in the world displayed by Geographic Information System maps. The European respiratory journal, 54(1), 1900610. https://doi.org/10.1183/13993003.006102019

Cazzola, M., MacNee, W., Martinez, F. J., Rabe, K. F., Franciosi, L. G., Barnes, P. J., Brusasco, V., Burge, P. S., Calverley, P. M., Celli, B. R., Jones, P. W., Mahler, D. A., Make, B., Miravitlles, M., Page, C. P., Palange, P., Parr, D., Pistolesi, M., Rennard, S. I., Rutten-van Mölken, M. P., ... European Respiratory Society Task Force on outcomes of COPD (2008). Outcomes for COPD pharmacological trials: from lung function to biomarkers. The European respiratory journal, 31(2), 416-469. https://doi.org/10.1183/09031936.00099306

Cheng, A. L., Hsu, C. H., Lin, J. K., Hsu, M. M., Ho, Y. F., Shen, T. S., Ko, J. Y., Lin, J. T., Lin, B. R., Ming-Shiang, W., Yu, H. S., Jee, S. H., Chen, G. S., Chen, T. M., Chen, C. A., Lai, M. K., Pu, Y. S., Pan, M. H., Wang, Y. J., Tsai, C. C., ... Hsieh, C. Y. (2001). Phase I clinical trial of curcumin, a chemopreventive agent, in patients with high-risk or pre-malignant lesions. Anticancer research, 21(4B), 2895-2900

Criner, G. J., Celli, B. R., Brightling, C. E., Agusti, A., Papi, A., Singh, D., Sin, D. D., Vogelmeier, C. F., Sciurba, F. C., Bafadhel, M., Backer, V., Kato, M., Ramírez-Venegas, A., Wei, Y. F., Bjermer, L., Shih, V. H., Jison, M., O'Quinn, S., Makulova, N., Newbold, P., ... TERRANOVA Study Investigators (2019). Benralizumab for the Prevention of COPD Exacerbations. The New England journal of medicine, 381(11), 10231034. https://doi.org/10.1056/NEJMoa1905248

Global Initiative for Chronic Obstructive Lung Disease. (2020) Global Strategy for the diagnosis, management, and prevention of chronic obstructive pulmonary disease (2020 Report). Retrieved from: https://goldcopd.org/wp-content/uploads/2019/11/GOLD-2020REPORT-ver1.0wms.pdf

Guarascio, A. J., Ray, S. M., Finch, C. K., \& Self, T. H. (2013). The clinical and economic burden of chronic obstructive pulmonary disease in the USA. ClinicoEconomics and outcomes research: CEOR, 5, 235-245. https://doi.org/10.2147/CEOR.S34321

Jones P. W. (1994). Quality of life, symptoms and pulmonary function in asthma: long-term treatment with nedocromil sodium examined in a controlled multicentre trial. Nedocromil Sodium Quality of Life Study Group. The European respiratory journal, 7(1), 55-62.

Ko, F. W., Chan, K. P., Hui, D. S., Goddard, J. R., Shaw, J. G., Reid, D. W., \& Yang, I. A. (2016). Acute exacerbation of COPD. Respirology (Carlton, Vic.), 21(7), 1152-1165. https://doi.org/10.1111/resp.12780

Lao, C. D., Ruffin, M. T., 4th, Normolle, D., Heath, D. D., Murray, S. I., Bailey, J. M., Boggs, M. E., Crowell, J., Rock, C. L., \& Brenner, D. E. (2006). Dose escalation of a curcuminoid formulation. BMC complementary and alternative medicine, 6, 10. https://doi.org/10.1186/1472-6882-6-10

Luber, R. P., Rentsch, C., Lontos, S., et al.(2019). Turmeric Induced Liver Injury: A Report of Two Cases. Case Reports in Hepatology, 2019, 1-4. doi:10.1155/2019/6741213

Meja, K. K., Rajendrasozhan, S., Adenuga, D., Biswas, S. K., Sundar, I. K., Spooner, G., Marwick, J. A., Chakravarty, P., Fletcher, D., Whittaker, P., Megson, I. L., Kirkham, P. A., \& Rahman, I. (2008). Curcumin restores corticosteroid function in monocytes exposed to oxidants by maintaining HDAC2. American journal of respiratory cell and molecular biology, 39(3), 312-323. https://doi.org/10.1165/rcmb.2008-00120C

Panahi, Y., Ghanei, M., Hajhashemi, A., \& Sahebkar, A. (2016). Effects of Curcuminoids-Piperine Combination on Systemic Oxidative Stress, Clinical Symptoms and Quality of Life in Subjects with Chronic
Pulmonary Complications Due to Sulfur Mustard: A Randomized Controlled Trial. Journal of dietary supplements, 13(1), 93105.https://doi.org/10.3109/19390211.2014.952865

Prasad, S., Tyagi, A. K., \& Aggarwal, B. B. (2014). Recent developments in delivery, bioavailability, absorption and metabolism of curcumin: the golden pigment from golden spice. Cancer research and treatment : official journal of Korean Cancer Association, 46(1), 2-18. https://doi.org/10.4143/crt.2014.46.1.2

Rabe, K. F., Martinez, F. J., Ferguson, G. T., Wang, C., Singh, D., Wedzicha, J. A., Trivedi, R., St Rose, E., Ballal, S., McLaren, J., Darken, P., Aurivillius, M., Reisner, C., Dorinsky, P., \& ETHOS Investigators (2020). Triple Inhaled Therapy at Two Glucocorticoid Doses in Moderate-to-VerySevere COPD. The New England journal of medicine, 383(1), 35-48. https://doi.org/10.1056/NEJMoa1916046

Sasaki, H., Sunagawa, Y., Takahashi, K., Imaizumi, A., Fukuda, H., Hashimoto, T., Wada, H., Katanasaka, Y., Kakeya, H., Fujita, M., Hasegawa, K., \& Morimoto, T. (2011). Innovative preparation of curcumin for improved oral bioavailability. Biological \& pharmaceutical bulletin, 34(5), 660665. https://doi.org/10.1248/bpb.34.660

Shoba, G., Joy, D., Joseph, T., Majeed, M., Rajendran, R., \& Srinivas, P. S. (1998). Influence of piperine on the pharmacokinetics of curcumin in animals and human volunteers. Planta medica, 64(4), 353-356. https://doi.org/10.1055/s-2006-957450

U.S. Department of Health and Human Services (2017). Common Terminology Criteria for Adverse Events (CTCAE) Version 5.0 [PowerPoint slides]. Retrieved from https://ctep.cancer.gov/protocolDevelopment/electronic_applicatio ns/docs/CTCAE_v5_Quick_Reference_5x7.pdf 